\title{
Phospholipase $A_{2}$ inhibition as potential therapy for inflammatory skin diseases
}

\section{“...the findings and considerations ... provide clear evidence of the merits of considering the control of the production of inflammatory lipid mediators, including lysoPL by inhibition of phospholipase $A_{2}$ activities for the treatment of skin inflammatory/allergic disease."}

\section{KEYWORDS: leukotriene "lipid mediator $\approx$ lysophospholipid $\approx$ phospholipase $A_{2}$ - prostaglandin $=$ skin allergy $=$ skin inflammation}

Inflammatory skin diseases are diverse (including allergies, autoimmune diseases, ulcers and so on) with different etiologies (hereditary, environmental or infectious), but their treatment is often similar. By far the most common treatment is the use of topical corticosteroids. Decades of usage have demonstrated their potency but they are also associated with a wide range of side effects [1] and patient-aversion to their usage. The quest for novel anti-inflammatory drugs (particularly topical ones) that can provide a safer alternative to steroids has understandably been undertaken by the pharmaceutical industry. One such potential target is phospholipase $\mathrm{A}_{2}\left(\mathrm{PLA}_{2}\right)$, a family of enzymes that has been studied extensively for over three decades.

$\mathrm{PLA}_{2}$ catalyze the hydrolysis of phospholipids to produce lysophospholipids and free fatty acids (in particular arachidonic acid) [1], thereby initiating the production of numerous lipid mediators that play key roles in the diverse inflammatory/allergic diseases. PLA 2 enzymes are categorized into different classes according to cellular localization, molecular weight, disulfide bond pattern, calcium dependence, sequence and $\mathrm{pH}$ of activity. The main groups are the secreted PLA 2 (sPLA $)$, cytosolic PLA $2\left(\mathrm{cPLA}_{2}\right)$ and Ca-independent $\mathrm{PLA}_{2}\left(\mathrm{iPLA}_{2}\right)$ [1]. Each group, in turn, has a number of isoforms.

sPLA ${ }_{2}$ s are a low-molecular-weight (average $14 \mathrm{kD}$ ) group of enzymes (16 isoforms, denoted types IA-XIV), characterized by at least six disulfide bonds, an absolute requirement for histidine in the active site and a dependence on $\mathrm{mM} \mathrm{Ca}^{2+}$ concentration. In general, $\mathrm{SPLA}_{2} \mathrm{~s}$ are mainly involved in the pathophysiology of inflammatory diseases and have long been considered the 'inflammatory enzymes' [2], although some sPLA 2 isoforms have been reported to also have a protective, anti-inflammatory potential. The most well-established physiological role of ${ }_{s P L A}$ is as an antimicrobial agent $[1,2]$.

The $\mathrm{CPLA}_{2} \mathrm{~s}$, Group IV, are larger than the sPLA $_{2} \mathrm{~s}(61-114 \mathrm{kD})$ with a different pattern of disulfide bonds. Their active site contains a serine/aspartic acid dyad and they require $\mu \mathrm{M}$ $\mathrm{Ca}^{2+}$ for activity. Group IVA cPLA 2 is specific for arachidonic acid in the sn 2 position, although other members of this group - cPLA Group IVB and cPLA ${ }_{2}$ Group IVC - show no specificity and cPLA 2 Group IVD is specific for linoleic acid. $\mathrm{CPLA}_{2}$ h have been shown to play a role in physiological/homeostatic processes such as cell cycle, gestation and female reproduction, but are also involved in various pathological conditions such as atherosclerosis, neurodegeneration and allergy.

The Ca-independent PLA ${ }_{2}$ s (Group VIA-F) do not show any preference for fatty acid at the sn 2 site and are considered to be mainly involved in membrane remodeling/homeostasis. In addition, they play a role in processes connected with reactive oxygen species metabolism and recently a role for iPLA $\mathrm{A}_{2}$ in obesity has been proposed.

By releasing free fatty acids from membrane phospholipids, $\mathrm{PLA}_{2}$ s provide the source for two large families of inflammatory lipid mediators. When the fatty acid is arachidonic acid, it is metabolized into the numerous eicosanoids by a number of downstream parallel enzymatic pathways, mainly the cyclooxygenases, producing the prostaglandins and thromboxanes, and the lipoxygenases, producing leukotrienes and epoxins, among others $[1,3]$.

While most of the attention and ample research has been focused on these eicosanoids [4], lysophospholipids, the second product of $\mathrm{PLA}_{2}$ activity, have been largely ignored, despite clear evidence of their role in inflammation. In

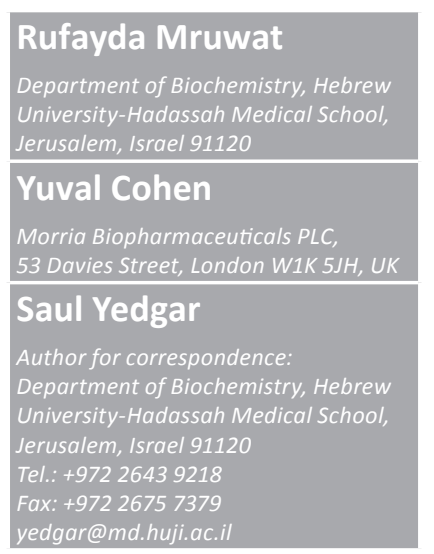

Future
Medicine $\mathrm{fS}$ 
general, lysophospholipids induce activation and extravasation of leukocytes, activate histamine secretion by mast cells, are precursors of the potent inflammatory mediator PAF, induce tissue damage, act as a growth factor and induce proliferation of smooth muscle and cancer cells, and tumor metastases. Moreover, while eicosanoids production is also regulated by a series of enzymatic pathways downstream from PLA (cyclooxygenases, lipoxygenases and so on), lysoPL are directly produced from PL by PLA. Yet, the control of PLA activities for the treatment of inflammatory/allergic diseases has been primarily based on their role in eicosanoid production, while their role in lysoPL production has practically been overlooked [5].

\section{"The quest for novel anti-inflammatory drugs (particularly topical ones) that can provide a safer alternative to steroids has understandably been undertaken by the pharmaceutical industry."}

In the skin, different $\mathrm{PLA}_{2}$ s have been found to be involved in processes relating to skin physiology and pathology, and this subject was extensively addressed in our previous review [4]. As noted above, the attention to the role of PLA $\mathrm{P}_{2}$ in skin function has been based primarily on the eicosanoid involvement in skin inflammatory/allergic conditions. It has long been reported that dermatitis - both atopic and allergic contact - as well as psoriasis, are associated with elevated production and accumulation of eicosanoids such as LTB 4 and 12-hydroxyeicosatetranoic [4], implying elevation of $\mathrm{PLA}_{2}$ activities. In this article, we would like to expand on the role of lysoPL as direct products of PLA 2 action in skin physiology and pathology.

For example, lysophosphatidylcholine (lysoPC)stimulating leukocyte activation is elevated in psoriatic skin and appears to contribute to the induction of inflammatory and immunological processes occurring in psoriatic skin lesions [6]. LysoPC also mediates melanocyte dendricity $[7,8]$, T-lymphocyte chemotraction [9] and histamine release from mast cells [10], inducing erythrema and edema $[9,10]$. In addition, lysoPC is a precursor of PAF, a potent inflammatory mediator found in psoriatic skin [4]. Lysophosphatidic acid (LPA) activates histamine release from mast cells and skin fragments [11], and activates $\mathrm{Cl}$ current activity in skin fibroblasts, which is considered a hallmark of scleroderma skin fibroblasts [12].

On the other hand, lysoPL, especially LPA, appear to also have beneficial effects on skin. For example, LPA, by induction of the migration of human fibroblasts and stimulation of cell growth, facilitates wound healing [13]; topical application of LPA was found to promote wound healing [14]. LysoPL, particularly LPA, showed activities that facilitate skin wetting [15] and hair growth. Topical administration of small amounts of lysoPC to nude mice was shown to have bactericidal and antiviral activity without damaging skin structure [16].

Given its role in inflammation, the control of $\mathrm{PLA}_{2}$, especially sPLA $\mathrm{P}_{2}$, has long been sought as a therapeutic strategy in the treatment of numerous inflammatory diseases [1,2], but rarely for treating skin diseases. The prospect of PLA 2 inhibition for treating skin inflammation has been demonstrated in previous studies using sPLA $\mathrm{A}_{2}$ inhibitors. In a clinical study by Ingber $e t a l$., topical application of a cream containing a cell-impermeable sPLA ${ }_{2}$ inhibitor, designed and synthesized in our lab, was shown to be effective in treating those suffering from allergic contact dermatitis [17]. In a study by Otuki et al., another sPLA inhibitor suppressed sPLA ${ }_{2}$ activity in $\mathrm{HaCat}$ and primary human keratinocytes stimulated with 12-O-tetradecanoylphorbol-3-acetate (TPA) concomitantly with $\mathrm{PGE}_{2}$ production [18]. In this study, a topical application of this inhibitor attenuated experimental TPA-induced dermatitis in mice [18]. These studies provide experimental evidence to support the potential of inhibiting the activity of PLA $\mathrm{s}_{2}$, especially secretory PLA, as a therapeutic approach in the treatment of inflammatory dermatological conditions.

\section{"While most of the attention and ample research has been focused on these eicosanoids, lysophospholipids, the second product of the phospholipase $A_{2}$ activity, have been largely ignored, despite clear evidence of their role in inflammation."}

In conclusion, the findings and considerations summarized above provide clear evidence of the merits of considering the control of the production of inflammatory lipid mediators, including lysoPL by inhibition of PLA $\mathrm{P}_{2}$ activities for the treatment of skin inflammatory/allergic disease. However, $\mathrm{PLA}_{2}$-generated lipid mediators, especially lysoPL, are also involved in healthy skin physiological functions, such as barrier permeability, skin $\mathrm{pH}$ and membrane PL remodeling $[4,19]$, and some $\mathrm{PLA}_{2} \mathrm{~s}$, especially the intracellular ones, have essential homeostatic roles [1,2]. In addition, it is well known that specific lipid mediators and PLA 2 isoenzymes might play 
different, sometimes opposing, roles in different organs and pathologies [1,5]. Thus, as with many drugs, systemic inhibition of their activity would necessarily induce adverse effects, pointing to the advantage of topical treatments with limited systemic penetration (as expected from cell-impermeable sPLA $\mathrm{i}_{2}$ inhibitors). The specific $\mathrm{PLA}_{2}$ isoenzyme to be targeted, which skin conditions it plays a role in and the specific methods of administration, have yet to be explored.

\section{References}

1 Murakami M, Taketomi Y, Miki Y, Sato H, Hirabayashi T, Yamamoto K. Recent progress in phospholipase $\mathrm{A}(2)$ research: from cells to animals to humans. Prog. Lipid Res. 50(2), 152-192 (2011).

2 Yedgar S, Cohen Y, Shoseyov D. Control of phospholipase A2 activities for the treatment of inflammatory conditions. Biochim. Biophys. Acta 1761, 1373-1382 (2006).

3 Ariel A and Serhan CN. Resolvins and protectins in the termination program of acute inflammation. Trends Immunol. 28(4), 176-183 (2007).

4 Dan P, Rosenblat G, Yedgar S. Phospholipase A2 activities in skin physiology and pathology. Eur. J. Pharmacol. 691(1-3), 1-8 (2012).

5 Yedgar S, Krimsky M, Cohen Y, Flower RJ. Treatment of inflammatory diseases by selective eicosanoid inhibition: a doubleedged sword? Trends Pharmacol. Sci. 28, 459-464 (2007).

6 Ryborg A K, Gron B, Kragballe K. Increased lysophosphatidylcholine content in lesional psoriatic skin. Br. J. Dermatol. 133(3), 398-402 (1995).

7 Scott GA, Jacobs SE, Pentland AP. sPLA2-X stimulates cutaneous melanocyte dendricity and pigmentation through a lysophosphatidylcholine-dependent

\section{Financial \& competing interests disclosure}

$S$ Yedgar is on the board of, served as a scientific officer for and has shares in Morria Biopharmaceuticals. Y Cohen is employed by and has shares in Morria Biopharmaceuticals. The authors have no other relevant affliations or financial involvement with any organization or entity with a financial interest in or financial conflict with the subject matter or materials discussed in the manuscript apart from those disclosed.

No writing assistance was utilized in the production of this manuscript.

mechanism. J. Invest. Dermatol. 126(4), 855-861 (2006).

8 Scott GA, Arioka M, Jacobs SE. Lysophosphatidylcholine mediates melanocyte dendricity through PKCzeta activation. J. Invest. Dermatol. 127(3), 668-675 (2007).

9 Ryborg AK, Deleuran B, Sogaard H, Kragballe K. Intracutaneous injection of lysophosphatidylcholine induces skin inflammation and accumulation of leukocytes. Acta Derm. Venereol. 80(4), 242-246 (2000).

10 Moreno JJ, Ferrer X, Ortega E, Carganico G. PLA2-induced oedema in rat skin and histamine release in rat mast cells. Evidence for involvement of lysophospholipids in the mechanism of action. Agents Actions 36(3-4), 258-263 (1992).

11 Hashimoto T, Ohata H, Honda K. Lysophosphatidic acid (anti) induces plasma exudation and histamine release in mice via anti receptors. J. Pharmacol. Sci. 100(1), 82-87 (2006).

12 Rhim JH, Jang IS, Kwon ST, Song KY, Yeo EJ, Park SC. Activation of wound healing in aged rats by altering the cellular mitogenic potential. J. Gerontol. A Biol. Sci. Med. Sci. 65(7), 704-711 (2010).

13 Demoyer JS, Skalak TC, Durieux ME. Lysophosphatidic acid enhances healing of acute cutaneous wounds in the mouse. Wound Repair Regen. 8(6), 530-537 (2000).
14 Balazs L, Okolicany J, Ferrebee M, Tolley B, Tigyi G. Topical application of the phospholipid growth factor lysophosphatidic acid promotes wound healing in vivo. Am. J. Physiol. Regul. Integr. Comp. Physiol. 280(2), R466-R472 (2001).

15 Yahagi S, Koike M, Okano Y, Masaki H. Lysophospholipids improve skin moisturization by modulating of calciumdependent cell differentiation pathway. Int. J. Cosmet. Sci. 33(3), 251-256 (2011).

16 Uchida Y, Ogawa T, Ohta M, Kondo M, Takada S, Yamamura M. Penetration of lysophosphatidylcholine into the dermis. J. Dermatol. 18(9), 523-527 (1991).

17 Ingber A, Cohen Y, Krimsky M, Yedgar S. A novel treatment of contact dermatitis by topical application of phospholipase A2 inhibitor: a double-blind placebo-controlled pilot study. Int. J. Immunopathol. Pharmacol. 20, 191-195 (2007).

18 Otuki MF, Schwob O, Prudente AS et al. Attenuation of experimental TPA-induced dermatitis by acetylenic acetogenins is associated with inhibition of PLA2 activity. Eur. J. Pharmacol. 672(1-3), 175-179 (2011).

19 Fluhr JW, Behne MJ, Brown BE et al. Stratum corneum acidfication in neonatal skin: secretory phospholipase A2 and the sodium/hydrogen antiporter-1 acidify neonatal rat stratum corneum. J. Invest. Dermatol. 122, 320-329 (2004). 